\section{PicoGmeter, a custom-made fluorometer for the quantification of dsDNA by PicoGreen ${ }^{\circledR}$ fluorescence}

\author{
Geoffrey G. Schofield \\ Tulane University Health Sciences Center, New Orleans, LA, USA
}

BioTechniques 37:778-782 (November 2004)

The accurate determination of DNA concentration is essential for many processes in molecular biology and physiology and includes both gel- and cuvette-based methods. The recently introduced fluorescent dye, PicoGreen ${ }^{\circledR}$, has several advantages over other methods because it is sensitive and specific for double-stranded DNA (dsDNA). The dye is excited at $480 \mathrm{~nm}$ and emits at $520 \mathrm{~nm}$ when bound to dsDNA. This report describes the construction and use of PicoGmeter, a simple, inexpensive, fixed-wavelength fluorometer suitable for measuring PicoGreen fluorescence. PicoGmeter employs a blue light emitting diode (LED) for excitation and a photodiode to measure fluorescence. When compared to a commercially available instrument, PTI DeltaScan ${ }^{\circledR}$, the PicoGmeter performed admirably. Calibration curves for both instruments were superimposeable. Moreover, there was no significant difference between concentrations of DNA estimated by both instruments. A Bland and Altman analysis revealed that the PicoGmeter was equivalent to the PTI DeltaScan for estimating dsDNA concentration by the PicoGreen method. This simple, inexpensive, battery-operated fluorometer will allow investigators to employ the PicoGreen method without incurring the cost of purchasing a spectrofluorometer.

\section{INTRODUCTION}

The accurate determination of double-stranded DNA (dsDNA) concentration is essential for many processes in molecular biology and physiology. Several methods exist to determine dsDNA concentration and include both gel- and cuvette-based methods. Probably the most commonly used method relies on the fact that DNA absorbs ultraviolet (UV) light with peak optical density (OD) at 260 $\mathrm{nm}$ and provides an estimate of both DNA concentration and quality (1). Recently, several fluorescent dyes have become available that increase fluorescence emission when bound to DNA (2-4). These reagents are sensitive and specific for dsDNA. One of these agents, PicoGreen ${ }^{\circledR}$ (Molecular Probes, Eugene, OR, USA) (3) is excited at 480 $\mathrm{nm}$ and emits at $520 \mathrm{~nm}$ when bound to dsDNA.

This report describes the construction and use of PicoGmeter, a simple, inexpensive, fixed-wavelength fluorometer suitable for measuring PicoGreen fluorescence. The estimated cost of the components (batteries not included) is around U.S. $\$ 20.00$, and construction requires little electronic experience.

\section{MATERIALS AND METHODS}

PicoGmeter was constructed in a small circuit enclosure (Figure 1A). The fluorescence cuvette holder, which was found in departmental surplus, was separated from the battery compartment by the measuring circuit (Figure 1B). A blue light emitting diode (LED; Agilent Technologies, Santa Clara, CA, USA) was used for excitation, and a photodiode (PerkinElmer Optoelectronics, Fremont, CA, USA) was used to measure emission. The LED and photodiode were attached to the cuvette holder by center drilling two pieces of acrylic rod to accommodate the LED and photodiode, which were then attached to the optical windows with hot glue. The cuvette holder is shown on the left of the circuit enclosure (Figure 1A) and on the circuit diagram as a dashed rectangle (Figure 1C). Note the orthogonal arrangement of the LED and photodiode. Although the data presented in this report were collected using a "real" cuvette holder, a serviceable holder incorporating space for an emission filter was made from aluminum and required only simple milling and drilling operations as follows. A 1/2 in wide, 1/2 in deep slot was milled into the front face of a piece of aluminum measuring 1 in $\times 1$ in $\times 2$ in. A 3/16 in hole, to accommodate the LED, was then drilled through the edge of the block, 1/4 in above the bottom, to intersect the center of the $1 / 2$ in slot. This hole was then widened with a $5 / 16$ in drill from the opposite side to a depth of $13 / 4 \mathrm{in.}$ A 3/16 in hole to accommodate the photodiode was drilled through the back face of the block, $1 / 4$ in above the bottom, which also intersected the center of the $1 / 2$ in slot. This hole was then widened front and back with the $1 / 2$ in end mill to accommodate an emission filter and to place the photodiode close to the cuvette. A piece of black plastic was glued to the front face to hold the cuvette in place. The resulting cuvette holder should be blackened to reduce stray light either by anodizing (http://www.focuser.com/ atm/anodize/anodize.html) or with a durable flat black paint. A drawing of the cuvette holder is available from the author.

The measuring circuit was adapted from a negative bias, current-to-voltage converter circuit described on the web (Figure 1, B and C; http://www. centrovision.com/tech2.htm) and was constructed using wire wrap technology. Other circuit prototyping options such as the experimenter socket from RadioShack or presoldered circuit boards (Vector Electronics \& Technology, North Hollywood, CA, USA) may be used. A CA3130 operational amplifier (http://www.arky. ru/audio/sprav/datasheets/ca3130.pdf) was used for the current-to-voltage converter because it can be powered from a single supply allowing battery operation. Two 9-volt (V) batteries, an on/off switch, and a $15-\mathrm{V}$ positive voltage regulator (NTE 968; NTE Electronics, Bloomfield, NJ, USA) provided a convenient power supply.

The $1 \mathrm{G} \Omega$ feedback resistor used in the measuring circuit (Figure 1, B and C) can be obtained from Electronic Sensor + Resistor GmbH (Höhen- 
kirchen, Germany) for the cost of shipping and handling. The output of the fluorescence measurement circuit was connected to a digital voltmeter (DVM) for routine measurements and to an oscilloscope and computer-based data acquisition system during initial testing. The connection to the recording equipment was made via a Bayonet Neil-Concelman (BNC) connector and cable. The BNC cable required a standard BNC-to-banana plug connector for connection to the DVM.
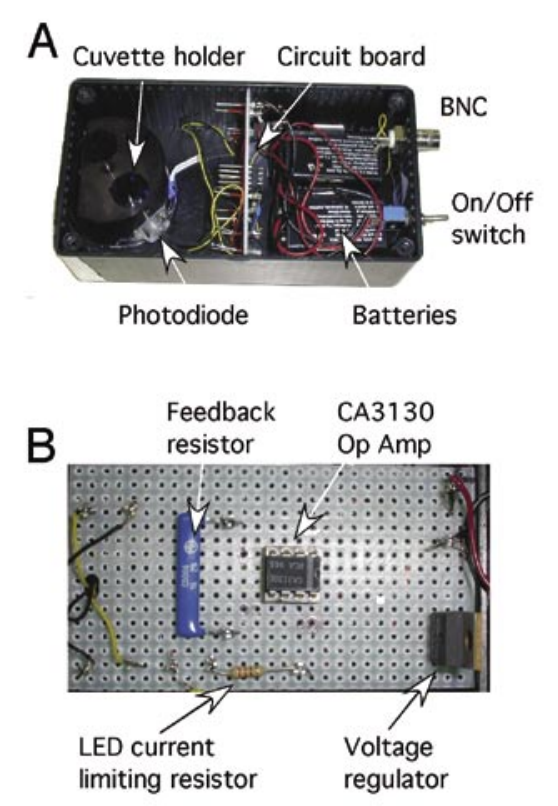

C

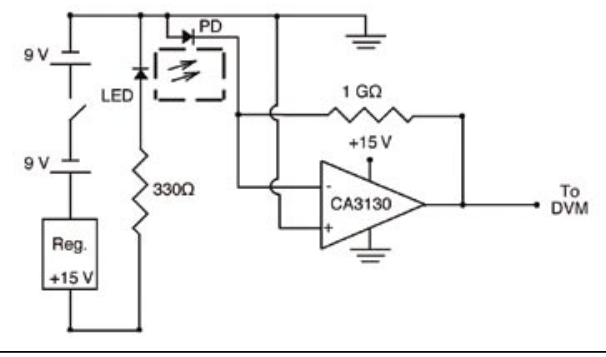

Figure 1. PicoGmeter and circuit diagram. (A) Internal arrangement of the PicoGmeter. The cuvette holder is on the left, the circuit board is placed in the middle of the enclosure, and the batteries are placed on the right. The on/off switch and Bayonet Neil-Concelman (BNC) connector output are also shown on the right of the enclosure. (B) Circuit layout shows the simplicity of the measuring circuit. (C) Circuit diagram showing the power supply and current-to-voltage converter. The cuvette holder is shown as a dashed rectangle. Note the orthogonal arrangement of the light emitting diode (LED) and photodiode (PD). Op Amp, operational amplifier; DVM, digital voltmeter.
All measurements were made using a Fluke $^{\circledR}$ Series 77 DVM obtained from Newark Electronics (Chicago, IL, USA). However, any DVM capable of measuring 0 to $15 \mathrm{~V}$ with a $10 \mathrm{mV}$ sensitivity could be used. The circuit enclosure was shielded and grounded to minimize electrical noise resulting from the high gain provided by the feedback resistor. Under these conditions, the unfiltered (10 kHz bandwidth) noise output was $90 \mathrm{mV}$ root mean square (RMS). All data were processed with the IGOR $\operatorname{Pro}^{\oplus}$ analysis package (Wavemetrics, Lake Oswego, OR, USA). Output voltages from the PicoGmeter were entered manually into IGOR, while the binary data from the PTI DeltaScan ${ }^{\circledR}$ (Photon Technology International, Lawrenceville, $\mathrm{NJ}$, USA) file were entered using routines provided by Dr. Stephen Ikeda (NIAAA/National Institutes of Health, Rockville, MD, USA). All fluorescence measurements were made using acrylic cuvettes (Sarstedt, Newton, NC, USA) after diluting DNA in PicoGreen $(2.5 \mu \mathrm{L} / \mathrm{mL})$ in TE $(10$ mM TRIS, 1 mM EDTA) buffer. Table 1 shows a list of parts and suggested vendors.

\section{RESULTS AND DISCUSSION}

The performance of the PicoGmeter was initially tested by determining the concentration of an "unknown" dsDNA sample. The results were compared to those obtained from a dual-monochrometer-based fluorometer (PTI DeltaScan), an Eppendorf ${ }^{\circledR}$ Biophotometer (OD $260 \mathrm{~nm}$; Brinkmann, Westbury, NY, USA), or from an ethidium bromide-stained MassRuler $^{\circledR}$ DNA Ladder (Fermentas, Hanover, MD, USA) in an agarose gel. Figure 2 shows calibration curves obtained from the PTI DeltaScan (filled squares) and PicoGmeter (open circles). The curves were constructed by serial dilution of the $\lambda$ phage DNA standard provided with the PicoGreen kit. Each point represents the mean of 4-6 separate samples. The error bars represent the standard deviation (SD) of the means. The inset shows the low end of the calibration curves on an expanded scale. Data from the PTI DeltaScan were extremely linear and reproducible, whereas the data from the PicoGmeter show deviation from linearity at lower DNA concentrations that would lead to an overestimation of DNA concentration. Both calibration curves in Figure 2 are from blank-subtracted values. Because an emission filter was not employed in the PicoGmeter, the blank value was approximately $26 \%$ of the raw $200 \mathrm{ng} / \mathrm{mL}$ reading, whereas the blank value from the PTI DeltaScan was only $2 \%$ of the raw $200 \mathrm{ng} / \mathrm{mL}$ reading. The high background of the PicoGmeter was not due to particulates in the solution because filtered $(0.2 \mu \mathrm{m})$ Ultrapure $^{\circledR}$ water also produced a high background. Thus, the high background likely originated from light scatter off the surfaces within the cuvette holder. The lower detection limit of the PicoGmeter was $<3 \mathrm{ng} / \mathrm{mL}$ since this concentration produced a signal that differed significantly from that of blank samples $(P<0.05$, paired Student's $t$ test $)$. The upper limit of detection $(450 \mathrm{ng} / \mathrm{mL})$ of the PicoGmeter was estimated by extrapolating the calibration curve to maximum output voltage. The lower detection limit of the PTI DeltaScan was not determined but is expected to be much lower than $3 \mathrm{ng} / \mathrm{mL}$ since the calibration curve at the lower limit test remained linear (Figure 2, inset). The upper detection limit $(250 \mathrm{ng} / \mathrm{mL})$ of the PTI DeltaScan was also estimated by extrapolating the calibration curve to the point of saturation of the photon counter. The dynamic ranges of the PicoGmeter $(<3$ to $450 \mathrm{ng} / \mathrm{mL}$ ) and the PTI DeltaScan $(<3$ to $250 \mathrm{ng} / \mathrm{mL})$ were considerably less than that of the PicoGreen dye, which has a dynamic range of $25 \mathrm{pg} / \mathrm{mL}$ to $1000 \mathrm{ng} / \mathrm{mL}$.

Table 2 shows the concentration of the unknown plasmid DNA sample determined by fluorometry, OD 260, or by comparison to the MassRuler. There was no statistical difference between the estimates from the two fluorometers or the MassRuler estimate. However, the concentration of the crude alkaline lysis DNA derived from the OD 260 
Table 1. Parts List

\begin{tabular}{|llll|}
\hline Part & Manufacturer & Distributer & Price \\
\hline $\begin{array}{l}\text { Blue LED } \\
\text { HLMP-CB15 }\end{array}$ & Agilent Technologies & Newark Electronics & $\$ 1.96$ \\
$\begin{array}{l}\text { photodiode VTB1113 } \\
\text { CA3130 operational amplifier }\end{array}$ & PerkinElmer Optoelectronics & Newark Electronics & $\$ 4.36$ \\
15 V positive & Harris Semiconductor & Newark Electronics & $\$ 1.20$ \\
$\begin{array}{l}\text { voltage regulator } \\
\text { NTE } 968\end{array}$ & NTE Electronics & Newark Electronics & $\$ 1.75$ \\
1 G $\Omega$ resistor & & & Shipping and Handling \\
CS 600 1G 2\% & Electronic Sensor + Resistor & Electronic Sensor + Resistor & \\
Micro on/off switch & GmbH & GmbH & $\$ 1.25$ \\
$\begin{array}{l}\text { Circuit enclosure } \\
(6 \text { in } \times 3 \text { in } \times 2 \text { in })\end{array}$ & JDR Microdevices & RDR Microdevices & $\$ 3.75$ \\
$\begin{array}{l}\text { Manufacturers and distributors are suggested, but with the exception of the feedback resistor, most parts may be obtained from any commercial electronics sup- } \\
\text { ply house. V, volts; LED, light emitting diode. }\end{array}$ & \\
\hline
\end{tabular}

Table 2. DNA Concentration Estimated by Fluorescence, OD 260 nm, and by Densitometry Using the MassRuler DNA Ladder

\begin{tabular}{|c|c|c|c|c|}
\hline & PTI DeltaScan & PicoGmeter & OD $260 \mathrm{~nm}$ & MassRuler \\
\hline DNA ng/ $\mu \mathrm{L}$ & $63.8 \pm 1.1$ & $64.8 \pm 2.1$ & $655.5 \pm 10.0^{a}$ & $57.4 \pm 1.3$ \\
\hline \multicolumn{5}{|c|}{$\begin{array}{l}\text { Fluorescence measurements from the PTI DeltaScan and PicoGmeter were taken from the same sam- } \\
\text { ples after 1:1999 dilution in PicoGreen }(2.5 \mu \mathrm{L} / \mathrm{mL}) \text {. Optical density (OD) } 260 \text { measurements were made } \\
\text { in a } 70-\mu \mathrm{L} \text { sample volume after a } 3: 67 \text { dilution. The MassRuler sample }(2 \mu \mathrm{L}) \text { was added to } 23 \mu \mathrm{L} \text { of } 1 \times \\
\text { loading buffer ( } 1.7 \text { mM Tris- } \mathrm{HCl}, \mathrm{pH} 7.6,0.005 \% \text { bromophenol blue, } 10 \% \text { glycerol, } 10 \mathrm{mM} \text { EDTA) made } \\
\text { up in nuclease-free water. DNA concentrations were determined from a grayscale image of the gel by } \\
\text { densitometry using National Institutes of Health (NIH) Image Software V1.62. Each DNA concentration } \\
\text { estimate is shown as the } \bar{x} \pm \text { SEM of four samples. aP }<0.01 \text { by one-way analysis of variance followed by } \\
\text { the Student-Newman-Keuls post hoc test. }\end{array}$} \\
\hline
\end{tabular}

method was overestimated by $10-$ fold, despite a 260:280 ratio of 1.85 . Subjecting the sample to a plasmid DNA cleanup procedure (QIAprep ${ }^{\circledR}$ Spin Miniprep Kit; Qiagen, Valencia

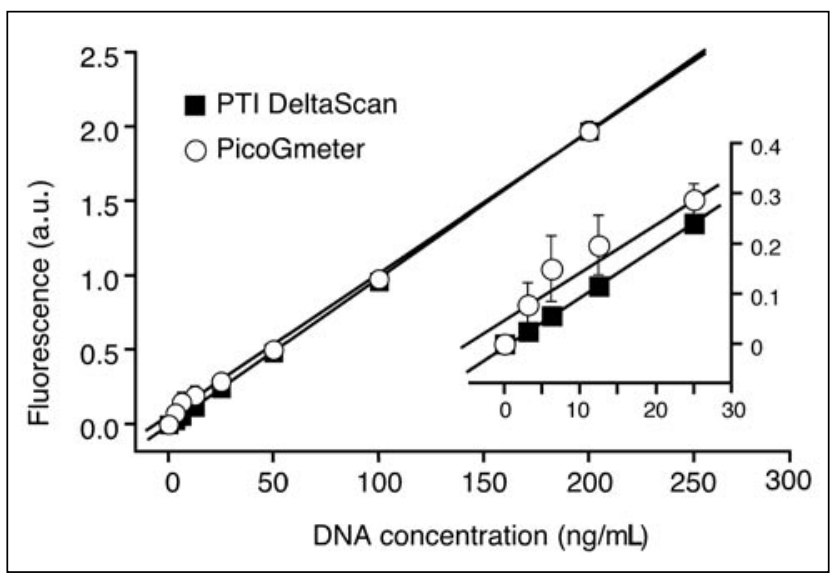

Figure 2. Calibration curves for PicoGreen from the PicoGmeter (open circles) and the PTI DeltaScan (filled squares). The calibration curve for the custom fluorometer has been scaled to the $200 \mathrm{ng} / \mathrm{mL}$ point of the PTI DeltaScan. The inset shows the low end of the calibration curves on an expanded scale. Each point is the mean of 4-6 samples. The error bars are \pm 1 standard deviation. The solid lines represent linear regression fits to the data. The samples were prepared by serial dilution of the standard $\lambda$ phage DNA supplied with the PicoGreen kit. a.u., arbitrary units.
CA, USA) reduced the discrepancy between concentrations estimated by fluorometry and OD 260 to 3-fold, whereas samples isolated by a QIAGEN Plasmid Midiprep Kit were in error by 2 -fold. On the other hand, concentration estimates of the dsDNA standard provided in the PicoGreen Kit ( $\lambda$ phage DNA) were similar using all four methods (data not shown). Although RNA, sodium dodecyl sulphate (SDS), and nucleotides are known to interfere with concentration estimates (1) based on the OD 260, the magnitude of these errors is indeterminate, and it is not widely appreciated that such errors can occur despite a "good" 260:280 ratio.

The excellent agreement of the unknown DNA sample concentration and the near superimposition of the calibration curves suggest that the PicoGmeter and PTI DeltaScan produce comparable results. To more rigorously compare the performance of the PicoGmeter and PTI DeltaScan, a Bland and Altman $(5,6)$ analysis was performed over a range of DNA concentrations (0-250 $\mathrm{ng} / \mathrm{mL})$. The paired concentration estimates plotted with the line of identity (Figure 3A) suggest excellent agreement between the two methods. The plot of the difference between the samples versus the mean of the samples strengthens this conclusion. Most of the points lie between \pm 1 SD of the differences. Although Bland and Altman analysis confirms that the PicoGmeter tends to overestimate DNA concentrations near the limit of detection, the methods are on the whole equivalent.

To test the battery life of the PicoGmeter, the output voltage was set to maximum by inserting a reflector into the cuvette holder at an angle of $45^{\circ}$. Under these conditions, the output voltage remained unchanged for $7 \mathrm{~h}$ before beginning to decline.

A common problem with fluorescent indicators is photobleaching, where the apparent quantum yield declines with continued excitation. However, photobleaching was not appreciable with the PicoGreen assay because the fluorescence intensity of a $100-\mathrm{ng} / \mathrm{mL}$ 


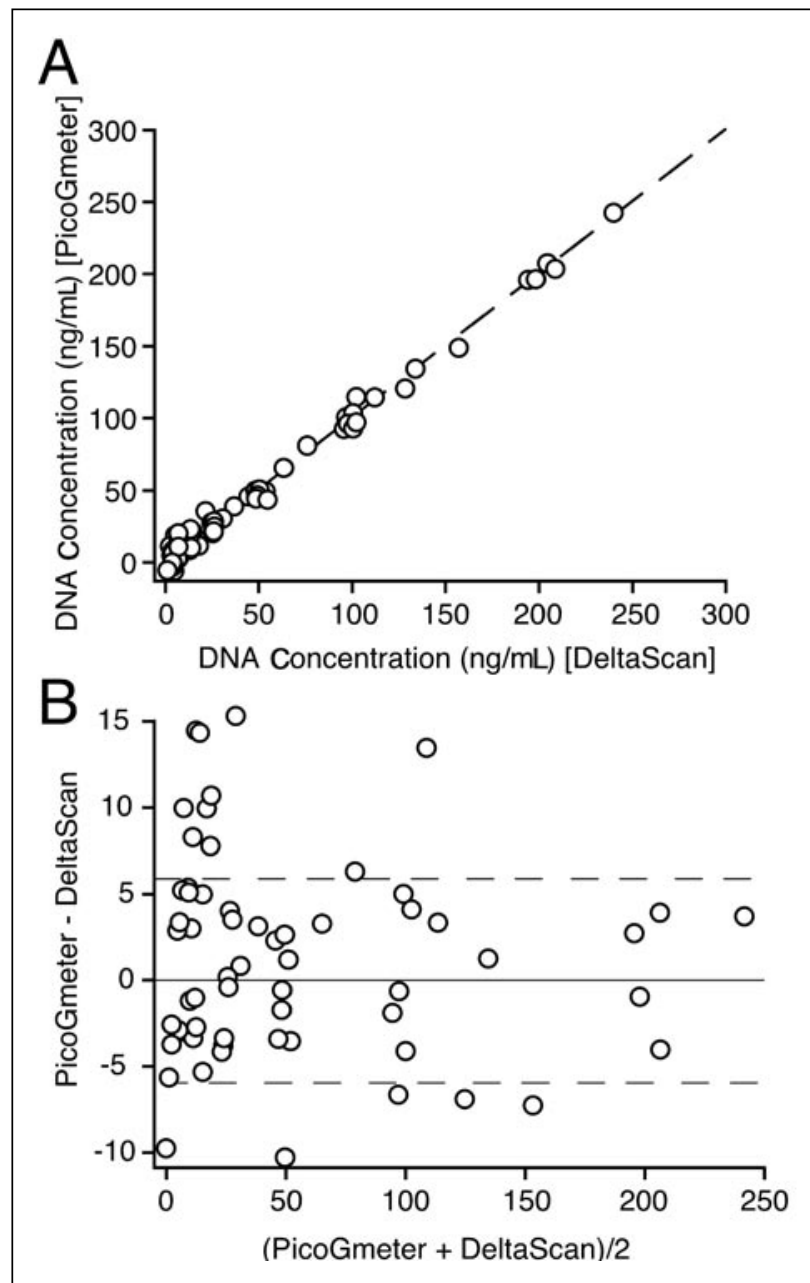

Figure 3. Bland and Altman analysis to test the equivalence of the PicoGmeter and the PTI DeltaScan. (A) Plot of DNA concentration estimates derived from PicoGmeter verses concentration estimates derived from the PTI DeltaScan. The solid line is the line of identity. (B) Plot of the mean differences of the paired samples versus the mean of the paired samples. The dotted lines represent \pm 1 standard deviation of the differences.

solution declined by only $5 \%$ during $15 \mathrm{~min}$ of continued excitation (data not shown). Moreover, because only a small sample volume is illuminated, the effects of photobleaching could be minimized when necessary by mixing the sample.

There are several possible modifications that may improve the performance and versatility of the PicoGmeter. Incorporation of a suitable emission filter may decrease the background fluorescence and increase signal-tonoise ratio. However, preliminary experiments with an emission filter similar to a Wratten ${ }^{\circledR} \# 8$ high-pass filter (Eastman Kodak, Rochester, NY, USA) produced only a marginal increase in signal-to-noise ratio, whereas a 520-nm narrow bandpass $[10 \mathrm{~nm}$ frequency width half maximum (FWHM)] filter (Edmund Industrial Optics, Barrington, NJ, USA) decreased the signal below the level of detection (data not shown). Because the performance of the PicoGmeter without an emission filter was nearly equivalent to the PTI DeltaScan, further experiments with emission filters were not pursued. A green or red LED with narrow spectral output may enable other dyes with suitable excitation spectra to be employed. Other photodiodes might be used as the detector. For example, a photodiode with a greater current output may allow the use of a lower feedback resistor. Finally, placing the LED and photodiode on opposite sides of the cuvette holder would allow the PicoGmeter to measure optical density.

In summary, this report describes the construction of the PicoGmeter, a simple, inexpensive, battery-operated fluorometer suitable for the determination of dsDNA concentration using the fluorescent dye, PicoGreen. The blue LED at the heart of the fluorometer has a peak output that coincides closely with the excitation peak of PicoGreen. The performance of PicoGmeter compares well to commercially available products costing several thousand dollars and will allow the impecunious investigator to employ the PicoGreen method without incurring the cost of purchasing a spectrofluorometer.

\section{ACKNOWLEDGMENTS}

Many thanks to Stephen Ikeda, M.D., Ph.D., (NIAAA/NIH) for IGOR Pro routines, data acquisition software, and for comments on the manuscript. This work was supported in part by a grant from the Louisiana Board of Regents Millennium Trust Health Excellence fund (2001-06)-07 to the Tulane Hypertension and Renal Center of Excellence.

\section{COMPETING INTERESTS STATEMENT}

The author declares no competing interests.

\section{REFERENCES}

1.Ausubel, F.M., R. Brent, R.E. Kingston, D.D. Moor, J.G. Seidman, J.A. Smith, and K. Struhl (Eds.) 1988. Quantitation of DNA and RNA with absorption and fluorescence spectroscopy, Appendix 3D. In Current Protocols in Molecular Biology. John Wiley and Sons, New York.

2.Rye, H.S., S. Yue, D.E. Wemmer, M.A. Quesada, R.P. Haugland R.A. Mathies, and A.N. Glazer. 1992. Stable fluorescent complexes of double-stranded DNA with bisintercalating asymmetric cyanine dyes: properties and applications. Nucleic Acids Res. 20:2803-2812.

3.Singer, V.L., L.J. Jones, S.T. Yue, and R.P. Haugland. 1997. Characterization of PicoGreen reagent and development of a fluorescence-based solution assay for doublestranded DNA quantitation. Anal. Biochem. 249:228-238

4.Labarca, C. and K. Paigen. 1980. A simple, rapid, and sensitive DNA assay procedure. Anal. Biochem. 102:344-352.

5.Altman, D.G. and J. Bland. 1983. Measurement in medicine: the analysis of method comparison studies. Statistician 32:307-317.

6.Bland, J. and D.G. Altman. 1986. Statistical methods for assessing agreement between two methods of clinical measurement. Lancet 8:307-310

Received 17 May 2004; accepted 27 July 2004.

Address correspondence to Geoffrey G. Schofield, Department of Physiology, Tulane University Health Sciences Center, 1430 Tulane Ave., New Orleans, LA 70112, USA.e-mail: solar@tulane.edu 